\title{
Peripancreatic vascular involvement in patients with type 1 autoimmune pancreatitis
}

\author{
Meizi Li ${ }^{1 \#}$, Xiaoyin Bai ${ }^{1 \#}$, Kai Xu' ${ }^{2}, \mathrm{Xi} \mathrm{Wu}^{1}$, Tao Guo ${ }^{1}$, Qingwei Jiang ${ }^{1}$, Qiang Wang ${ }^{1}$, Shengyu Zhang ${ }^{1}$, \\ Yingyun Yang ${ }^{1}$, Yunlu Feng ${ }^{1}$, Aiming Yang ${ }^{1}$
}

${ }^{1}$ Department of Gastroenterology, State Key Laboratory of Complex Severe and Rare Diseases, Peking Union Medical College Hospital, Peking Union Medical College and Chinese Academy of Medical Sciences, Beijing, China; ${ }^{2}$ Department of Radiology, Peking Union Medical College Hospital, Beijing, China

Contributions: (I) Conception and design: M Li, X Bai, Y Feng, A Yang; (II) Administrative support: A Yang, X Wu; (III) Provision of study materials or patients: Q Jiang, T Guo, Q Wang, S Zhang, Y Yang, X Wu, K Xu, Y Feng, A Yang; (IV) Collection and assembly of data: X Bai, M Li; (V) Data analysis and interpretation: M Li, X Bai, Y Feng, A Yang; (VI) Manuscript writing: All authors; (VII) Final approval of manuscript: All authors.

"These authors contributed equally to this work.

Correspondence to: Professor Yunlu Feng, MD. Department of Gastroenterology, Peking Union Medical College Hospital, Endoscopy Center, No. 1 Shuaifuyuan Dongcheng District, Beijing 100730, China. Email: yunluf@icloud.com; Professor Aiming Yang, MD. Vice Chairperson of Chinese Society of Digestive Endoscopy, Chief of Department of Gastroenterology, Peking Union Medical College Hospital, Endoscopy Center, No. 1 Shuaifuyuan Dongcheng District, Beijing 100730, China. Email: yangam2020@126.com.

Background: Type 1 autoimmune pancreatitis (AIP) is the pancreatic manifestation of IgG4-related disease. However, this benign disease can result in the peripancreatic vascular involvement (PVI) on occasion, which increases the difficulty of diagnosis and treatment of this clinical entity as well as for differentiating it from pancreatic malignancies.

Methods: We retrospectively reviewed the information on demographics, clinical presentation, laboratory, imaging and endoscopic findings of 101 hospitalized patients with type 1 AIP treated in our department. All the patients were divided into non-PVI and PVI groups according to the first hospitalized medical data. Univariate and multivariate analyses were performed to analyse the potential predictive parameter(s) of PVI in AIP patients.

Results: Among the 101 type 1 AIP patients, 52 (51.5\%) exhibited PVI, with a male/female ratio 5.5:1. Their average age was $58.37 \pm 8.68$ years old. Univariate analysis revealed that the location of pancreatitis lesions, including the pancreatic tail $(\mathrm{P}=0.010)$, the presence of splenomegaly $(\mathrm{P}=0.001)$ and the white blood cell $(\mathrm{WBC})$ number in peripheral blood $(\mathrm{P}=0.020)$, were significantly associated with PVI. The location of pancreatitis lesions, including the pancreatic tail $(\mathrm{P}=0.023)$, and the presence of splenomegaly $(\mathrm{P}=0.010)$ were found to be independent predictors of the development of PVI by a multivariable regression analysis. A total of 18 out of 25 patients in PVI group who underwent corticosteroid treatment and no less than 6 months radiological follow-up showed improvement in vascular lesions, and no case exhibited exacerbation of PVI lesions during follow-up. Of 36 patients in non-PVI group who were followed up for no less than 6 months, only one case exhibited PVI.

Conclusions: This retrospective study demonstrated that type 1 AIP was associated with a high proportion of PVI. Pancreatic tail involvement and splenomegaly may predict the PVI in type 1 AIP. PVI lesions are reversible in a subset of patients.

Keywords: Autoimmune pancreatitis (AIP); peripancreatic vascular involvement (PVI); IgG4-related disease (IgG4-RD); splenomegaly; corticosteroid treatment

Submitted Feb 24, 2021. Accepted for publication May 13, 2021.

doi: $10.21037 / \mathrm{hbsn}-21-82$

View this article at: https://dx.doi.org/10.21037/hbsn-21-82 


\section{Introduction}

Autoimmune pancreatitis (AIP) is a distinct clinical entity with two distinguishable histopathological subtypes, namely, lymphoplasmacytic sclerosing pancreatitis (LPSP; type 1 AIP) and idiopathic duct-centric pancreatitis (IDCP; type 2 AIP). Type 1 AIP is the pancreatic manifestation of IgG4-related disease (IgG4-RD) (1). Vascular involvement can be one of the characteristic biological behaviours of AIP. However, there are few reports focusing on peripancreatic vascular involvement (PVI). Kamisawa et al. evaluated 17 AIP patients and found that angiographic abnormalities occurred in 54\% of cases in 2003 (2). Five years later, Takahashi et al. reported vascular involvement in 44\% (11/25) of AIP patients (3). And then Ishikawa et al. extended these findings in 2012; they observed PVI in $44 \%$ (24/54) of AIP patients using computed tomography (CT) scans and highlighted the long-term outcome of PVI as well (4). Subsequently, Matsubayashi et al. compared 46 patients with AIP to matched patients with chronic pancreatitis of alternative aetiologies and reported splenic vein involvement (evaluated by CT scan) in 67\% (31/46) of AIP cases (5). To our knowledge, our study has the largest sample size of type 1 AIP patients with PVI. We described the clinical characteristics of type 1 AIP patients in an attempt to explore the significant factors associated with PVI based on individual data. We present the following article in accordance with the STROBE reporting checklist (available at https://hbsn.amegroups. com/article/view/10.21037/hbsn-21-82/rc).

\section{Methods}

\section{Patients}

From January 2013 to December 2018, a consecutive total of 101 patients hospitalized at department of gastroenterology with type 1 AIP were enrolled in Peking Union Medical College Hospital (PUMCH), a tertiary academic hospital, in Beijing, China. To analyse the potential predictive parameter(s) of PVI in AIP patients, all the patients in this cohort were divided into non-PVI and PVI groups as medical data were collected. Details of all the patients were extracted from the medical record database for further review and analysis. The study was conducted in accordance with the Declaration of Helsinki (as revised in 2013). The study was approved by the Ethics Committee of PUMCH (No. S-K1449-1) and informed consent was taken from all individual participants.

\section{Diagnostic criteria}

We reviewed the medical data (including clinical, laboratory, radiological, endoscopic and histological features) of 101 patients. The diagnosis of AIP was made according to the Asian diagnostic criteria for AIP (6). Peri-pancreatic vasculature is defined as an entity of splenic, and superior mesenteric artery, along with portal, superior mesenteric, and splenic vein. Based on this, PVI was defined as the presence of encasement and stenosis/occlusion of peripancreatic vasculature and the accompanying lesions, such as peri-gastric collateral circulation formation, thrombosis or splenic infarction secondary to vascular involvement.

\section{Research approach}

We collected data on age at diagnosis, gender, and the following clinical characteristics: initial symptom(s), duration from symptom to diagnosis, smoking and alcoholism, comorbidity, disease course duration, clinical phenotype (with or without recurrent acute pancreatitis), and other organ involvement (OOI) (7). In terms of routine laboratory parameters, we collected detailed data from complete blood cell tests, hepatic and renal function analysis, coagulation, total serum amount of immunoglobulin (Ig), serum IgG4 and inflammatory index. In addition, we focused on the CT imaging features of the pancreatitis lesion (the pancreatic inflammatory lesion involved by the IgG4-RD), cyst formation induced by pancreatitis and specific peripancreatic vessels morphology (splenic and superior mesenteric vessels and portal venous system). Gastric, splenic portal and celiac varices were recorded elaborately.

Multidetector computed tomography (MDCT) examinations were performed on 128-row multi-detector CT scanners (Somatom Definition Flash, Siemens Healthinners, Forchheim, Germany). The scanning parameters were: tube voltage, $120 \mathrm{kVp}$; effective amperage settings, $150 \mathrm{mAs}$; gantry rotation time, $0.5 \mathrm{~s}$; table increment, $46.8 \mathrm{~mm}$ per rotation; matrix, $512 \times 512$. After unenhanced scan of the upper abdomen, non-ionic contrast material with $1.5 \mathrm{~mL} / \mathrm{kg}$ of body weight was injected intravenously at a rate of $4.0 \mathrm{~mL} / \mathrm{s}$. Bolus tracking was used, and pancreatic arterial phase, portal venous phase and delayed phase were initiated with a 7-, 60- and 120-s delay after aortic enhancement of $100 \mathrm{HU}$, respectively. Slice thickness for contrast-enhanced images was $1-3 \mathrm{~mm}$. The CT images were retrospectively reviewed by 2 radiologists 

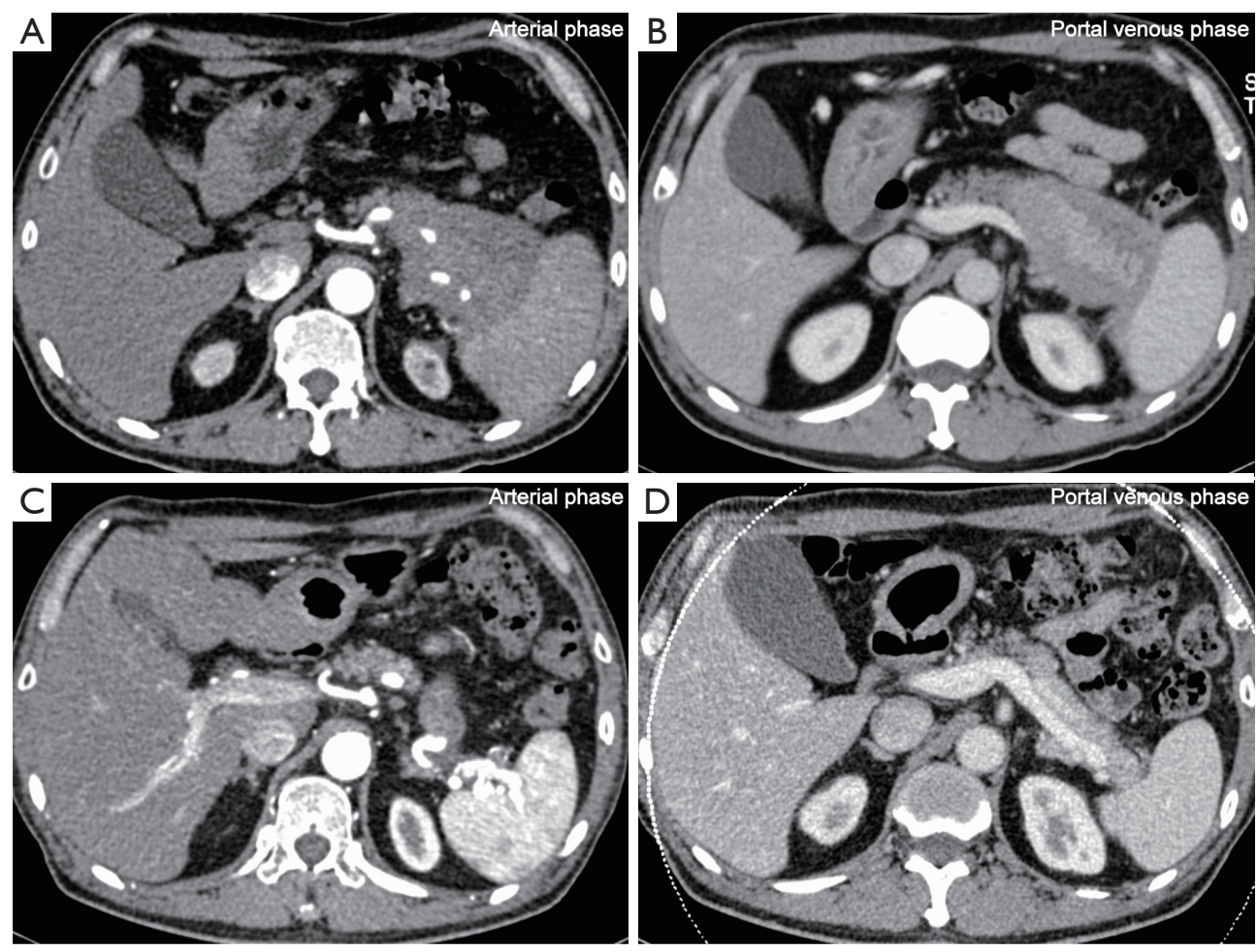

Figure 1 A 61-year-old male diagnosed as AIP with PVI. (A,B) Computed tomography images acquired at initial diagnosis. (C,D) Computed tomography images acquired after 1-year steroid therapy. $(\mathrm{A}, \mathrm{C})$ The present encasement of splenic artery showed improvement after 1-year steroid treatment. (B,D) The occlusion of splenic vein disappeared after 1-year steroid treatment. AIP, autoimmune pancreatitis; PVI, peripancreatic vascular involvement.

(15 and 6 years of experience in interpreting abdominal CT, respectively) on a local imaging workstation. Patient personal information, clinical and laboratory findings and treatment regimen were blinded to the reviewing radiologists.

\section{Statistical analysis}

All continuous variables are expressed as the mean \pm standard deviation or median, and the non-continuous variables are expressed as percentages. The parameters were compared using a bilateral $t$-test for continuous variables with normal distribution, Mann-Whitney $U$ test for abnormal distribution, and a chi-square test or Fisher's exact test with or without Bonferroni correction depending on the $\mathrm{P}$ value for non-continuous variables in two groups. A two-tailed $\mathrm{P}$ value $<0.05$ was considered statistically significant. The $95 \%$ confidence interval (CI) was calculated where appropriate. Multivariate logistic regression analysis was performed using the SPSS 23.0 software package (IBM
Corporation, Chicago, IL, USA).

\section{Results}

Among the 101 type 1 AIP patients, 52 (51.5\%) exhibited PVI (Figure 1A,1B). As shown in Table 1, of the specific vascular lesions observed, involvement of the splenic artery in 15 patients (encasement in 2/101, 2.0\%; stenosis in $13 / 101,12.9 \%$ ), involvement of the splenic vein was found in 43 patients (stenosis/occlusion in 42/101, 41.6\%; thrombosis in $1 / 101,1.0 \%$ ), stenosis of superior mesenteric artery in one patient, the stenosis/occlusion of the superior mesenteric-portal vein in $17.8 \%$ of patients (18/101) and stenosis/occlusion of the main portal vein in $6.0 \%$ patients (6/101). Peri-gastric collateral circulation formation was detected in 34 patients using MDCT (Figure S1A), and gastric varices with a red color sign were observed in 9 out of 35 patients who underwent an upper gastrointestinal endoscopy (Figure S1B). Multiple vascular involvement was found in $38.6 \%(39 / 101)$ of patients. 
Table 1 Prevalence of specific peripancreatic vascular involvement

\begin{tabular}{lc}
\hline PVI & Numbers of patients (\%) \\
\hline Splenic artery & $15 / 101(14.9)$ \\
Encasement & $2 / 101(2.0)$ \\
Stenosis & $13 / 101(12.9)$ \\
Splenic vein & $43 / 101(42.6)$ \\
Stenosis/occlusion & $42 / 101(41.6)$ \\
Thrombosis & $1 / 101(1.0)$ \\
Superior mesenteric artery stenosis & $1 / 101(1.0)$ \\
Superior mesenteric-portal vein & $18 / 101(17.8)$ \\
stenosis/occlusion & \\
Main portal vein stenosis/occlusion & $6 / 101(6.0)$ \\
Gastric varices with red color sign & $9 / 101(8.9)$ \\
Peri-gastric collateral circulation & $34 / 101(33.7)$ \\
formation & \\
Multiple vascular involvement & $39 / 101(38.6)$ \\
\hline PVI, peripancreatic vascular involvement.
\end{tabular}

$\mathrm{PVI}$, peripancreatic vascular involvement.

We analysed predictive variables for PVI in type 1 AIP patients without $(\mathrm{n}=49) /$ with $(\mathrm{n}=52)$ PVI. As shown in Table 2, univariate analysis revealed that the location of pancreatitis lesions, including the pancreatic tail $(\mathrm{P}=0.010)$, the presence of splenomegaly $(\mathrm{P}=0.001)$ and the white blood cell (WBC) number in peripheral blood $(\mathrm{P}=0.020)$, were significantly associated with PVI. The WBC number in peripheral blood $\left[(5.96 \pm 1.54) \times 10^{9}\right]$ was lower in cases with PVI than in cases without PVI $\left[(6.80 \pm 2.01) \times 10^{9}\right]$. There was no detectable difference in age, sex, smoking status, presence/absence of diabetes, presence/absence of cholelithiasis, presence/absence of recurrent acute pancreatitis, presence/absence of cyst formation, presence/ absence of diffuse pancreas swelling, presence/absence of OOI (7), serum amylase level, serum total bilirubin level, serum C-reactive protein level, serum IgG level, or serum IgG4 level in cases with PVI compared to cases without PVI. Significant differences were also not noted in the group with PVI or the location of pancreatitis lesions, including the pancreatic head and pancreatic body. Through multivariate analysis (Table 3), the location of pancreatitis lesions, including the pancreatic tail [odds ratio (OR), 3.06; 95\% CI, $1.17-8.02 ; \mathrm{P}=0.023$ ], and the presence of splenomegaly (OR, 8.23; 95\% CI, 1.67-40.51; $\mathrm{P}=0.010)$ were found to be independent predictors of the development of PVI.
On pre-treatment CT scans ( $\mathrm{n}=101), 44$ patients had a pancreatic mass (head in 32, tail in 11 and body in 1 ). Among these patients with atypical findings of the pancreas on radiographs (including low-density mass, pancreatic ductal dilatation, or distal atrophy) which are hints to pancreatic malignancies, 39 patients received EUSguided fine needle aspiration biopsy (EUS-FNA) to obtain histopathologic evidence to exclude pancreatic malignancies. The remaining 5 patients did not undergo endoscopic ultrasound due to the operational contraindications or personal wishes, but showed an excellent response to the corticosteroid therapy. At the end of follow-up, none of all patients enrolled had pancreatic adenocarcinoma or malignancies.

Corticosteroid treatment was administered to 46 of the 52 patients with vascular involvement. Among the remaining 6 patients, 2 patients were lost to follow-up, one patient had undergone the Whipple procedure in another hospital, one patient had contraindications for the use of corticosteroid treatment, and 2 patients refused corticosteroid therapy. Of the 46 patients, 38 patients underwent regular radiological follow-up. The median follow-up time was 10.5 months [interquartile range (IQR), 4.0-16.8 months]. Among the 25 patients followed up for no less than 6 months with MDCT, 18 patients showed improvement of PVI (Figure 1), and none of them exhibited exacerbation of PVI lesions during follow-up.

Of the 4 patients without corticosteroid treatment, one case had undergone the Whipple procedure and been cured. New vascular lesions were not detected in any of the remaining 3 patients; no change of both pancreatic and vascular lesions was seen in 2 of 3 patients 11 and 12 months after the first exam, and one patient exhibited spontaneous remission of both pancreatic and vascular lesions during a followed-up period of 43 months. All of the 49 patients without PVIs were followed up with corticosteroid treatment. Among 36 patients without PVI on pre-treatment $\mathrm{CT}$ scans who were followed up for no less than 6 months, only one case exhibited PVI.

\section{Discussion}

PVI is one of the characteristic biological behaviours of AIP, which hinders our diagnosis in differentiating AIP from pancreatic malignancies (5). In the present study, we identified potential risk factors for the development of PVI in patients with type 1 AIP, that is, the location of pancreatitis lesions, including the pancreatic tail, and the 
Table 2 Comparison of demographic and clinical characteristics of type 1 AIP patients with or without PVI

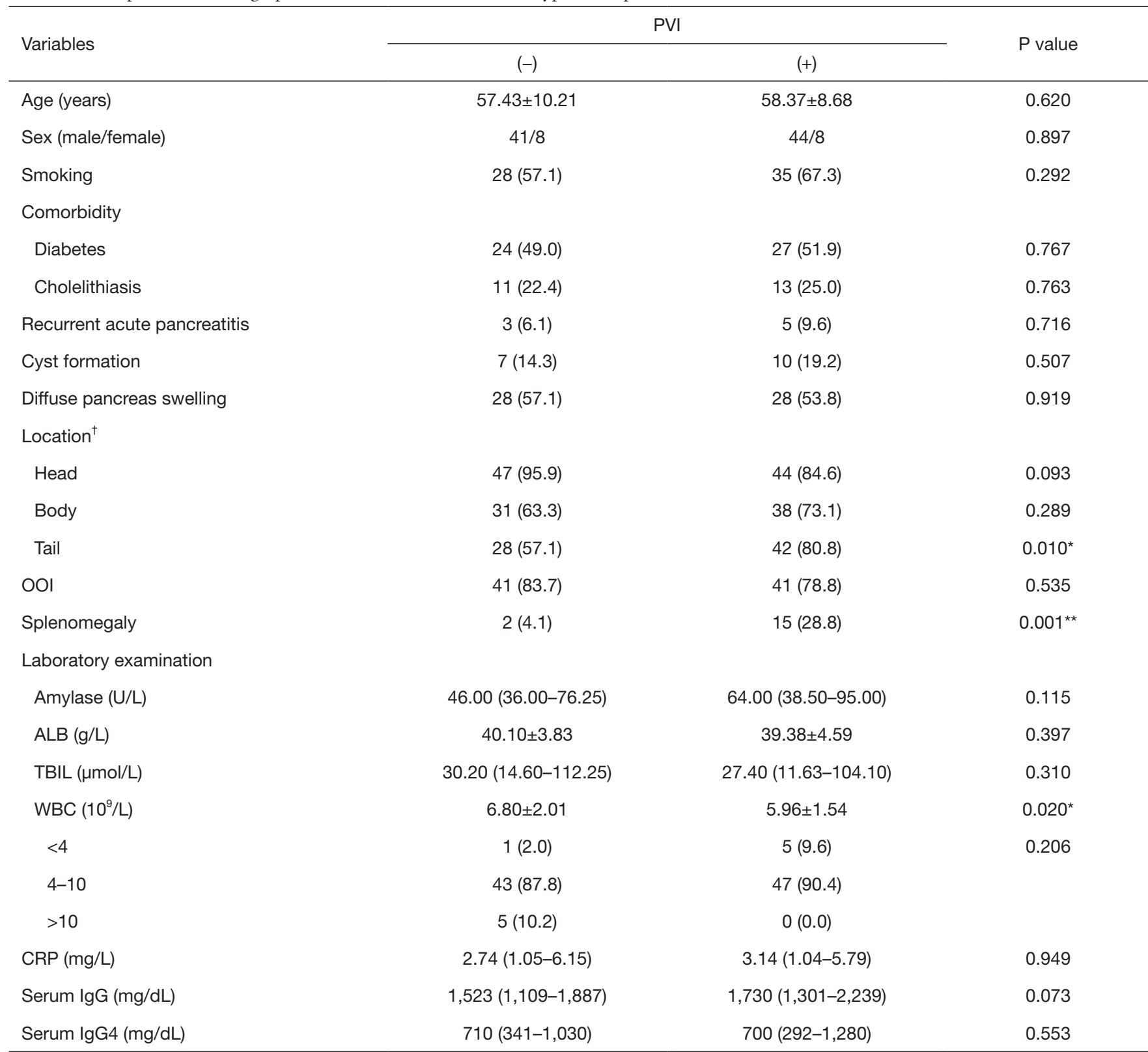

Value of age, WBC number shown as the mean \pm SD. ${ }^{\dagger}$, location of pancreatitis lesion included pancreatic head, pancreatic body or pancreatic tail; *, $\mathrm{P}<0.05$; ${ }^{\star}, \mathrm{P}<0.01$. Values of amylase, TBIL, CRP, IgG, and IgG4 are shown as the median (IQR). AIP, peripancreatic vascular involvement; PVI, peripancreatic vascular involvement; OOI, other organ involvement; ALB, albumin; TBIL, total bilirubin; WBC, white blood cell; CRP, C-reactive protein; IQR, interquartile range; SD, standard deviation.

presence of splenomegaly.

By comparison, different risk factors were observed in studies from other countries. Matsubayashi et al. reported that $\mathrm{SpV}$ involvement was predominantly recognized as diffuse type $(83.3 \%)$ rather than focal type $(57.1 \%)$ $(\mathrm{P}=0.06)$ (5). Ishikawa et al. used CT scans to observe the
PVI of any form and indicated diffuse pancreatic swelling of AIP as a relative risk factor for PVI compared to focal swelling (4). We attribute this difference to the techniques used in evaluating pancreatic and vascular involvement, geographic differences and the different sample sizes. And the results need to be confirmed in larger and prolonged 
Table 3 Multivariate regression analyses of risk factors associated with PVI in type 1 AIP patients

\begin{tabular}{lcc}
\hline Variables & OR $(95 \% \mathrm{Cl})$ & P value \\
\hline Location $^{\dagger}$ & & \\
Tail & $3.06(1.17-8.02)$ & $0.023^{\star}$ \\
Splenomegaly & $8.23(1.67-40.51)$ & $0.010^{\star}$ \\
WBC $\left(10^{9} / \mathrm{L}\right)$ & $0.77(0.59-1.00)$ & 0.051
\end{tabular}

${ }^{\dagger}$, location of the pancreatitis lesion including the pancreatic tail; *, $\mathrm{P}<0.05$. PVI, peripancreatic vascular involvement; AIP, peripancreatic vascular involvement; $\mathrm{Cl}$, confidence interval; OR odds ratio; WBC, white blood cell.

observational studies. Several of the findings on clinical presentation, including age, gender, prevalence of diabetes, prevalence of OOI and any of serum marker levels (serum IgG level, serum IgG4 level) in our series, were similar to those reported from Japan $(4,5)$.

The pathophysiology underlying the development of PVI in AIP is still under investigation. Previous studies detected that vascular involvement in AIP appears to be due to focal factors rather than a pre-existing hypercoagulable state $(4,8)$. This conclusion is in line with our findings. In the present study, we observed that the location of pancreatitis lesions, including the pancreatic tail, was significantly associated with PVI. In other words, vascular involvement was related to the site of pancreatic inflammation lesions. Ishikawa et al. found that the vascular lesions in AIP improved in most patients who underwent corticosteroid treatment (4). Matsubayashi et al. demonstrated that $\mathrm{SpV}$ stenosis reopened in all cases to some degree after corticosteroid therapy (5). In our study, $72.0 \%(18 / 25)$ patients with PVI who underwent corticosteroid treatment and follow-up for no less than 6 months with MDCT showed improvement of PVI, and no case exhibited exacerbation of PVI lesions during the follow-up period. These findings suggested that the possible mechanism of this vascular involvement was obliterative phlebitis (9-12) or simple oppression resulting from the inflammatory mass composed of lymphoplasmacytic aggregates and fibrosis, which responded to glucocorticoid therapy. To summarize, PVI in AIP has been related to many factors, which can be divided into mechanical force and inflammatory infiltration. The former includes compression by an enlarged pancreatic parenchyma or a pseudocyst or surrounding oedema. The latter refers to the extension of inflammatory changes from the pancreas and primary inflammatory process typical for an IgG4-related systemic disorder. The factors mentioned above can damage the vein directly or hinder venous flow through external compression. As a potent broad-spectrum anti-inflammatory drug, corticosteroid has a significant role in suppressing either simple compression resulting from the inflammatory mass composed of lymphoplasmacytic aggregates and fibrosis or direct inflammatory infiltration to peri-pancreatic vasculature.

We also found that the presence of splenomegaly was a risk factor for PVI. It is widely acknowledged that $\mathrm{SpV}$ obstruction can produce a distinctive leftsided, or "sinistral", portal hypertension that leads to the development of splenomegaly. However, we hold the opinion that this is not the only factor contributing to splenomegaly. Matsubayashi et al. documented that the splenic volume was larger for AIP cases without obvious $\mathrm{SpV}$ involvement $(144 \mathrm{~mL})$ than for age- and sex-matched controls $(91 \mathrm{~mL})$, and in these cases, the splenic volume decreased to $91 \%$ of the original state after corticosteroid therapy as well (5). These findings indicated that splenomegaly responsive to corticosteroids might be an autoimmune-associated phenomenon. Many autoimmune diseases, such as rheumatic arthritis and systemic lupus erythematosus, are known to involve splenomegaly, and corticosteroid treatment is effective for these diseases (13).

Overall, it is still too early to draw a definitive conclusion on the pathogenesis of PVI and AIP. Nevertheless, our study informs clinical physicians that they should be mindful that AIP is associated with a high prevalence of PVI. We believe that noninvasive radiological screening for PVI should be given strong consideration in patients with AIP to provide early intervention. Goto et al. reported 3 cases of AIP complicated by gastric varices and highlighted the prospect that corticosteroids reduce the development and potential reversibility of gastric varices in patients with AIP (14). Juarez et al. reported one case of AIP without concurrent splenic vein obstruction and indicated that early corticosteroid treatment directly reduces the risk of irreversible complications from splenic vein obstruction (15). On the basis of the finding of the present study, among 36 patients without PVI on pre-treatment CT scans who were followed up for no less than 6 months, only one case exhibited PVI. In addition, among the 25 patients with PVI who underwent corticosteroid treatment and regular radiological follow-up, 18 patients showed improvement of PVI, and no case exhibited exacerbation of PVI lesions during the follow-up period. These findings indicate that early intervention may prevent complications 
from vascular involvement to some extent, and PVI lesions are reversible, at least in a subset of patients.

There are some limitations of this study. Firstly, our current data may overestimate the incidence of PVI in patients with AIP due to a hospitalized population enrolled in this study. Secondly, we did not conduct an extensive long-term follow-up of the prognosis of PVI, and it is necessary to evaluate the merits and demerits of corticosteroid therapy by further investigation. Thirdly, this was a single-center cohort study, although it enrolled more than 100 patients with AIP, the results inferred from this study need be validated in a multicenter study further. Lastly, the present investigation was observational, and in addition to defining the pathogenesis and factors that determine vascular involvement, further prospective researches on this should be investigated.

In conclusion, this retrospective study demonstrated that AIP is associated with a high prevalence of PVI. The location of pancreatitis lesions, including the pancreatic tail and the presence of splenomegaly, were identified as potential risk factors for PVI in AIP. Corticosteroid treatment may prevent complications from vascular involvement to some extent, and PVI lesions are reversible, at least in a subset of patients. Future multi-institutional prospective investigations are warranted to provide additional evidence for pathophysiology, and the research results should be incorporated into clinical practice.

\section{Acknowledgments}

The authors would like to thank Dr. Huadan Xue from Department of Radiology, Peking Union Medical College Hospital, and Dr. Dongsheng Wu, Dr. Jiaming Qian, Dr. Hong Yang, and Dr. Yamin Lai from Department of Gastroenterology, Peking Union Medical College Hospital for their assistance.

Funding: This work was supported by National Natural Science Foundation of China, Joint Fund Project (grant No. U20A600), National Key R\&D Program of China (grant No. 2020YFC2002702), National Key Technology Research and Development Program (grant No. 2016YFC1302802) and Beijing Municipal Science and Technology Project (grant No. Z181100001618013).

\section{Footnote}

Reporting Checklist: The authors have completed the STROBE reporting checklist. Available at https://hbsn. amegroups.com/article/view/10.21037/hbsn-21-82/rc

Data Sharing Statement: Available at https://hbsn. amegroups.com/article/view/10.21037/hbsn-21-82/dss

Conflicts of Interest: All authors have completed the ICMJE uniform disclosure form (available at https://hbsn. amegroups.com/article/view/10.21037/hbsn-21-82/coif). The authors have no conflicts of interest to declare.

Ethical Statement: The authors are accountable for all aspects of the work in ensuring that questions related to the accuracy or integrity of any part of the work are appropriately investigated and resolved. The study was conducted in accordance with the Declaration of Helsinki (as revised in 2013). The study was approved by the Ethics Committee of Peking Union Medical College Hospital (No. S-K1449-1) and informed consent was taken from all individual participants.

Open Access Statement: This is an Open Access article distributed in accordance with the Creative Commons Attribution-NonCommercial-NoDerivs 4.0 International License (CC BY-NC-ND 4.0), which permits the noncommercial replication and distribution of the article with the strict proviso that no changes or edits are made and the original work is properly cited (including links to both the formal publication through the relevant DOI and the license). See: https://creativecommons.org/licenses/by-nc-nd/4.0/.

\section{References}

1. Uchida K, Okazaki K. Clinical and pathophysiological aspects of type 1 autoimmune pancreatitis. J Gastroenterol 2018;53:475-83.

2. Kamisawa T, Egawa N, Nakajima H, et al. Clinical difficulties in the differentiation of autoimmune pancreatitis and pancreatic carcinoma. Am J Gastroenterol 2003;98:2694-9.

3. Takahashi N, Fletcher JG, Fidler JL, et al. Dual-phase CT of autoimmune pancreatitis: a multireader study. AJR Am J Roentgenol 2008;190:280-6.

4. Ishikawa T, Itoh A, Kawashima $\mathrm{H}$, et al. Peripancreatic vascular involvements of autoimmune pancreatitis. J Gastroenterol Hepatol 2012;27:1790-5.

5. Matsubayashi H, Uesaka K, Kanemoto H, et al. Reduction of splenic volume by steroid therapy in cases with autoimmune pancreatitis. J Gastroenterol 2013;48:942-50. 
6. Otsuki M, Chung JB, Okazaki K, et al. Asian diagnostic criteria for autoimmune pancreatitis: consensus of the Japan-Korea Symposium on Autoimmune Pancreatitis. J Gastroenterol 2008;43:403-8.

7. Rispens T, Ooijevaar-de Heer P, Bende O, et al. Mechanism of immunoglobulin G4 Fab-arm exchange. J Am Chem Soc 2011;133:10302-11.

8. Raina A, Yadav D, Krasinskas AM, et al. Evaluation and management of autoimmune pancreatitis: experience at a large US center. Am J Gastroenterol 2009;104:2295-306.

9. Kobayashi G, Fujita N, Noda Y, et al. Lymphoplasmacytic sclerosing pancreatitis forming a localized mass: a variant form of autoimmune pancreatitis. J Gastroenterol 2007;42:650-6.

10. Zen Y, Nakanuma Y. IgG4-related disease: a cross-sectional study of 114 cases. Am J Surg Pathol 2010;34:1812-9.

11. Notohara K, Burgart LJ, Yadav D, et al. Idiopathic chronic

Cite this article as: Li M, Bai X, Xu K, Wu X, Guo T, Jiang Q, Wang Q, Zhang S, Yang Y, Feng Y, Yang A. Peripancreatic vascular involvement in patients with type 1 autoimmune pancreatitis. HepatoBiliary Surg Nutr 2022;11(3):355-362. doi: 10.21037/hbsn-21-82 pancreatitis with periductal lymphoplasmacytic infiltration: clinicopathologic features of 35 cases. Am J Surg Pathol 2003;27:1119-27.

12. Kamisawa T, Egawa N, Nakajima H, et al. Extrapancreatic lesions in autoimmune pancreatitis. J Clin Gastroenterol 2005;39:904-7.

13. Fishman D, Isenberg DA. Splenic involvement in rheumatic diseases. Semin Arthritis Rheum 1997;27:141-55.

14. Goto N, Mimura J, Itani T, et al. Autoimmune pancreatitis complicated by gastric varices: a report of 3 cases. World J Gastroenterol 2012;18:4228-32.

15. Juarez LA, Gupta RR, Ruhnke GW. Gastric varices and splenic vein obstruction during steroid treatment for autoimmune pancreatitis: A case report and literature review. Medicine (Baltimore) 2018;97:e11940. 